Reprod. Nutr. Dévelop., 1988, 28 Suppl. n 1, 183-184

\title{
Utilisation de l'urée comme marqueur de l'eau corporelle chez la vache, en comparaison avec l'eau lourde
}

M. DOREAU, C. M. GEERKEN $\left({ }^{1}\right)$, J. ROBELIN $\left({ }^{*}\right)$

Laboratoire de la Lactation.

(*) Laboratoire de la Production de viande, I.N.R.A., Theix, 63122 Ceyrat, France.

Summary. Urea dilution space was compared in 5 cows to deuterium oxide dilution space and to direct determination of body water after slaughter. Urea and deuterium oxide dilution spaces were respectively close to empty body water and total body water weights.

L'urée est utilisée depuis de nombreuses années pour estimer la quantité d'eau présente dans les organismes animaux, et par suite leur composition corporelle; elle n'a été que récemment utilisée chez la vache (Bartle, Males et Preston, 1983). Le présent essai a pour but de comparer l'espace de diffusion de l'urée avec celui d'un autre marqueur de l'eau corporelle, l'eau lourde, et avec des mesures directes de la quantité d'eau corporelle chez la vache.

Matériel et méthodes. Cinq vaches taries de race Limousine, pesant entre 583 et $640 \mathrm{~kg}$ (moyenne $623 \mathrm{~kg}$ ), et d'état d'engraissement moyen (4,0 à 5,0 sur une échelle de 0 à 10) ont été utilisées. Elles recevaient de l'ensilage de maïs à volonté et $500 \mathrm{~g}$ de MS de maïs grain. Quatre heures après la distribution du repas, de l'urée leur a été injectée dans la veine jugulaire à l'aide d'un cathéter, en solution à $40 \%$ dans du sérum physiologique, à raison de $200 \mathrm{mg}$ par $\mathrm{kg}$ de poids vif. L'injection, lente, a duré de 3 à $5 \mathrm{~min}$. Des échantillons de sang ont été prélevés par le cathéter $5,10,20,30,45,60,90,120,150,300$ et $600 \mathrm{~min}$ après la fin de l'infusion. L'accès aux aliments et l'abreuvement étaient libres pendant la durée de la mesure. La concentration en urée a été déterminée sur le plasma par une méthode colorimétrique utilisant l'uréase. L'espace de diffusion de l'urée a été calculé par 3 méthodes: $\left.1^{\circ}\right)$ Utilisation du modèle $C=E / f+\left(C_{0}-E / f\right) e^{-f t / V}$; $C, C o, E, V$, f t t représentent respectivement la concentration et la concentration initiale théorique, la vitesse d'entrée et l'espace de diffusion de l'urée, le flux d'élimination d'eau et le temps; $2^{\circ}$ ) et $3^{\circ}$ ) calcul à partir des concentrations en urée, respectivement 10 et 20 min après la fin de l'injection.

Deux jours après cette mesure, de l'eau lourde a été injectée aux vaches selon la technique décrite par Robelin (1982). L'analyse de la courbe de décroissance de concentration en eau lourde a été réalisée selon un modèle exponentiel à partir de prélèvements réalisés $6,8,30$ et $48 \mathrm{~h}$ après injection.

Le lendemain de la fin de la mesure de l'espace de diffusion de l'eau lourde, les vaches ont été abattues et entièrement disséquées. La teneur en eau des différents tissus a été mesurée. Ceci a permis de déterminer la quantité d'eau totale du corps vide et du contenu digestif, et, par addition, celle du corps entier. Cuba.

(1) Adresse actuelle : Instituto de Ciencia Animal - Apartado 24, San José de Las Lajas. Habana, 
Résultats et discussion. L'ajustement de la courbe de décroissance de la concentration de l'urée au modèle 1 de type exponentiel est très bon: les coefficients de détermination obtenus avec les points expérimentaux situés entre 20 et 600 min après l'injection sont compris entre 0,987 et 0,996. Ceci traduit que les flux d'entrée et de sortie d'urée sont des phénomènes réguliers. L'ajustement a été meilleur que dans un essai antérieur mené sur poulains (Geerken et al., 1988). Les méthodes 1 et 3 d'estimation de l'espace de diffusion de l'urée donnent des résultats voisins et proches de la quantité d'eau du corps vide: $324 \mathrm{~kg}$; ils s'en écartent en moyenne respectivement de -11 et $-14 \mathrm{~kg}$ (tabl. 1). Cette légère différence semble accréditer l'hypothèse d'une diffusion incomplète. En revanche, la méthode 2 sous-estime fortement l'eau du corps vide, alors que selon Preston et Kock (1973), la diffusion de l'urée dans l'organisme serait terminée $12 \mathrm{~min}$ après l'injection. La correspondance entre l'espace de diffusion de l'urée et l'eau du corps vide est cohérente avec l'absence de passage rapide d'urée dans le rumen mise en évidence par Bartle et Preston (1986). Dans cet essai, la faible teneur en azote du régime a probablement limité le transfert d'ammoniac vers le foie et donc la synthèse d'urée. La moyenne des écarts individuels entre les espaces de diffusion obtenus par les méthodes 1 et 3 et l'eau du corps vide est respectivement de 21 et $17 \mathrm{~kg}$. L'utilisation d'un modèle explicatif $n$ 'améliorerait donc pas la précision de la mesure. L'espace de diffusion de l'eau lourde est en moyenne inférieur de $4 \mathrm{~kg}$ à la quantité totale d'eau corporelle. La moyenne des écarts individuels entre ces deux estimations est de $6 \mathrm{~kg}$. Elle est donc plus faible que celle entre l'espace de diffusion de l'urée et l'eau du corps vide. Cependant, ces écarts n'entraînent pas de biais systématique important sur l'estimation de l'eau du corps vide. La précision moindre de l'urée par rapport à l'eau lourde est partiellement compensée par l'avantage d'un marquage de l'eau du corps vide.

TABL. 1. - Comparaison des espaces de diffusion de l'urée et de l'eau lourde et des quantités d'eau corporelle et du corps vide, en $\mathrm{kg}$.

\begin{tabular}{|c|c|c|c|c|c|c|}
\hline & \multicolumn{3}{|c|}{$\begin{array}{l}\text { Espace de diffusion } \\
\text { de l'urée }\end{array}$} & \multirow{2}{*}{$\begin{array}{c}\text { Espace de } \\
\text { diffusion } \\
\text { de l'eau } \\
\text { lourde }\end{array}$} & \multirow{2}{*}{$\begin{array}{c}\text { Eau } \\
\text { corporelle } \\
\text { totale }\end{array}$} & \multirow{2}{*}{$\begin{array}{c}\text { Eau du } \\
\text { corps } \\
\text { vide }\end{array}$} \\
\hline & Méthode 1 & Méthode 2 & Méthode 3 & & & \\
\hline Vache 1 & 275 & 248 & 282 & 383 & 387 & 318 \\
\hline Vache 2 & 321 & 260 & 326 & 381 & 392 & 339 \\
\hline Vache 3 & 324 & 242 & 314 & 385 & 388 & 332 \\
\hline Vache 4 & 323 & 284 & 324 & 386 & 381 & 334 \\
\hline Vache 5 & 322 & 243 & 306 & 354 & 363 & 296 \\
\hline Moyenne & 313 & 255 & 310 & 378 & 382 & 324 \\
\hline
\end{tabular}

Bartle S. J., Males J. R., Preston R. L., 1983. J. anim. Sci, 56, 410-417.

Bartle S. J., Preston R. L., 1986. J. anim. Sci., 63, 77-82.

Geerken C. M., Doreau M., Boulot S., 1988. Reprod. Nutr. Dévelop., 28, 257-263

Preston R. L., Kock S. W., 1973. Proc. Soc. exp. Biol. Med., 143, 1057-1061.

Robelin J., 1982. Reprod. Nutr. Dévelop., 22, 65-73. 